\title{
Estratégias Utilizadas para Melhorar a Qualidade dos Exames Citopatológicos
}

doi: https://doi.org/10.32635/2176-9745.RBC.2020v66n1.104

\author{
Strategies Used to Improve the Quality of Citopathological Examinations \\ Estrategias Utilizadas para Mejorar la Calidad de los Examenes Citopatológicos
}

Mackson Jardel Silva Santos'; Andrea Alves Ribeiro²

Resumo

Introdução: O exame citopatológico é utilizado para detecção precoce das lesôes precursoras do câncer do colo uterino. Objetivo: Avaliar os indicadores de qualidade de acordo com o Manual de Gestão da Qualidade para Laboratório de Citopatologia. Método: Verificaram-se os laudos das fichas de requisição dos exames citopatológicos do laboratório clínico da Pontifícia Universidade de Goiás (LC-PUC-Goiás) entre janeiro de 2013 e dezembro de 2017. Resultados: Do total de 6.809 diagnósticos, observaram-se 99,4\% (6.768/6.809) satisfatórios, sendo $91,3 \%$ (6.215/6.809) resultados negativos, 8,1\% (553/6.809) diagnósticos com anormalidades citológicas e 0,6\% (41/6.809) de exames insatisfatórios. O índice de positividade dos anos de 2013 a 2017 foram 10,5\%, 7,9\%, 8,6\%, 6,8\% e 5,3\%, respectivamente. O percentual de exames compatíveis com lesáo intraepitelial de alto grau (HSIL) entre os exames satisfatórios no ano de 2013 foi de 1,3\%; 2014: 1,0\%; 2015: 0,5\%; 2016: 0,6\%; e 2017: 0,7\%, resultados dentro do estabelecido, $\geq 0,4 \%$. A relação de células escamosas atípicas (ASC)/satisfatórios demostrou valores acima do estabelecido nos anos de 2013 com 6,8\% e 2015 com 6,1\%. Segundo o Manual de Gestão para Controle de Qualidade, espera-se que, no máximo, 4\% a 5\% de todos dos exames sejam classificados como ASC. Valores acima de 5\% necessitam de uma atenção diferenciada. Conclusáo: É de suma importância a educação continuada dos profissionais que participam de todas as etapas do processo, da fase pré-analítica à analítica, para que possíveis erros possam ser corrigidos e medidas preventivas tomadas para uma melhor qualidade na interpretação dos exames citopatológicos.

Palavras-chave: Biologia Celular; Controle de Qualidade; Neoplasias do Colo do Útero.

\section{Abstract}

Introduction: The cytopathological examination is used for early detection of cervical cancer precursor lesions. Objective: Evaluate the quality indicators according to the Quality Management Manual for the Cytopathology Laboratory. Method: The results of the request forms of cytopathological examinations of the Clinical Laboratory of the Pontifical University of Goiás (LC-PUC-Goiás) were verified between January 2013 and December 2017. Results: Of 6,809 diagnoses in total, 99.4\% $(6,768 / 6,809)$ were satisfactory, $91.3 \%(6,215 / 6,809)$ were negative, $8.1 \%$ $(553 / 6,809)$ presented cytological abnormalities and $0.6 \%(41 / 6,809)$ were unsatisfactory. The Positivity Index from 2013 to 2017 was $10.5 \%, 7.9 \%$, $8.6 \%, 6.8 \%$ and $5.3 \%$, respectively. The percentage of examinations with high-grade intraepithelial lesion (HSIL) among satisfactory examinations in 2013 was $1.3 \%$, in $2014,1.0 \%$, in $2015,0.5 \%$, in $2016,0.6 \%$ and in $2017,0.7 \%$, results within the established $\geq 0.4 \%$. The atypical squamous cells (ASC)/satisfactory ratio showed values above the standard figures in 2013 with 6.8\% and 2015 with 6.1\%. According to the Quality Control Management Manual, it is expected that, at the most, $4 \%$ to $5 \%$ of all exams be classified as ASC. Values above 5\% demand differentiated attention. Conclusion: It is of the utmost importance the continued education of professionals who participate in all stages of the process, from the preanalytical to the analytical phase, so that possible errors can be avoided and preventive measures taken for better quality interpretation of the cytopathological examinations.

Key words: Cell Biology; Quality Control; Uterine Cervical Neoplasms.

\section{Resumen}

Introducción: El examen citopatológico se utiliza para la detección temprana de lesiones precursoras de cáncer cervical. Objetivo: Evaluar los indicadores de calidad de acuerdo con el Manual de Gestión de Calidad para el laboratorio de citopatología. Método: Se verificaron los informes de los formularios de solicitud para los exámenes citopatológicos del Laboratorio Clínico de la Pontificia Universidad de Goiás (LC-PUC-Goiás), de enero de 2013 a diciembre de 2017. Resultados: Del total de 6.809 diagnósticos, se observarán 99,4\% (6.768/6.809) diagnósticos satisfactorios, 91,3\% (6.215/6.809) resultados negativos, 8,1\% (553/6.809) diagnósticos con anomalías citológicas y $0,6 \%(41 / 6.809)$ exámenes insatisfactorios. El índice de positividad de 2013 a 2017 fue de 10,5\%, 7,9\%, 8,6\%, 6,8\% y $5,3 \%$ respectivamente. El porcentaje de exámenes compatibles con lesiones intraepiteliales de alto grado (HSIL) entre los exámenes satisfactorios en 2013 fue de 1,3\%; 2014: 1,0\%; 2015: 0,5\%; 2016: 0,6\%; y 2017: 0,7\%, resultados dentro de lo establecido, $\geq 0,4 \%$. La relación células escamosas atípicas (ASC)/satisfactorio fue más alta que la establecida en 2013 con 6,8\% y 2015 con 6,1\%. Según el Manual de Gestión de Control de Calidad, se espera que un máximo del $4 \%$ al $5 \%$ de todos los exámenes se clasifiquen como ASC. Los valores superiores al 5\% requieren una atención diferente. Conclusión: Es de suma importancia la educación continua de los profesionales que participan en todas las etapas del proceso, desde la fase preanalítica hasta la analítica, para que se puedan corregir los posibles errores y se tomen medidas preventivas para una mejor calidad en la interpretación de los exámenes citopatológicos.

Palabra clave: Biología Celular; Control de Calidad; Neoplasias del Cuello Uterino.

\footnotetext{
${ }^{1}$ Escola de Ciências Médicas, Farmacêuticas e Biomédicas da Pontifícia Universidade Católica de Goiás (PUC-Goiás). Goiânia, (GO), Brasil. Orcid iD: https://orcid. org/0000-0002-8997-6039

${ }^{2}$ Escola de Ciências Médicas, Farmacêuticas e Biomédicas da PUC-Goiás. Goiânia, (GO), Brasil. Orcid iD: https://orcid.org/0000-0002-1692-7025

Endereço para correspondência: Mackson Jardel Silva Santos. Av. Universitária, 1.440 - Setor Universitário. Goiânia (GO), Brasil. CEP 74605-010.

E-mail:mackson.jardel@gmail.com
} 


\section{INTRODUÇÃO}

O câncer do colo uterino, exceto o câncer de pele não melanoma, é considerado o terceiro tumor mais frequente nas mulheres, sendo a quarta causa de morte por câncer da população feminina brasileira. Segundo o Instituto Nacional de Câncer José Alencar Gomes da Silva (INCA), no Brasil, a incidência estimada para o ano de 2018 é de 16.370 novos casos ${ }^{1}$. Este é causado pelo agente etiológico chamado papilomavírus humano (HPV) ${ }^{2}$.

As lesôes precursoras do câncer do colo uterino, nos diagnósticos citopatológicos, classificadas como lesôes intraepiteliais escamosas de alto grau (HSIL) e adenocarcinoma in situ (AIS) sáo provocadas pela infecção persistente por diversos tipos de HPV de alto risco oncogênico ${ }^{3}$. Existem fatores de risco que favorecem a infecção e/ou sua persistência tais como: início da atividade sexual precoce, número de parceiros, alta paridade, uso de contraceptivos orais e tabagismo, que facilitam sua infecção e persistência ${ }^{4}$.

Outro fator que pode contribuir para infecçáo pelo HPV é a zona de transformação, também chamada de junção escamocolunar, onde possui a presença do epitélio colunar e/ou metaplásico, aumentando as probabilidades de microtraumatismos e carcinogênese. Esse processo fisiológico é chamado ectopia, a área do colo do útero que mais possui capacidade de infecção, com maior número de células jovens em replicação e diferenciação, portanto maior possibilidade de encontrar as lesóes precursoras do câncer do colo uterino ${ }^{3,5}$.

A análise de uma amostra adequada é um indicador de qualidade fundamental, sendo assim uma amostra é considerada satisfatória quando contém células escamosas, glandulares e/ou metaplásicas; ou seja, da zona de transformação, em quantidades representativas e bem distribuídas, fixadas e coradas, que permita uma conclusão diagnóstica ${ }^{6}$.

A Organização Mundial da Saúde (OMS) recomenda a realização do exame citopatológico, de forma adequada, atingindo $80 \%$ de cobertura e realizado dentro dos parâmetros de qualidade. Nessas condiçôes, acredita-se que diminuem entre $60 \%$ a $90 \%$ as ocorrências do câncer do colo do útero ${ }^{7}$.

Para que se tenha um bom desempenho no rastreamento do câncer do colo do útero, é necessário que se façam métodos de alta sensibilidade, boa especificidade e que seja fácil a sua implementação. Nos últimos anos, o exame citopatológico vem sofrendo várias críticas em virtude da sua baixa sensibilidade ${ }^{8-10}$. As taxas dos falsos negativos variam entre $6 \%$ a $56 \%$, que podem ocorrer na fase pré-analítica e analítica ${ }^{11,12}$. Alguns desses motivos podem estar relacionados a problemas das coletas e outros nos processamentos e análises como o procedimento de coloração, no escrutínio e interpretação ${ }^{12}$.

Segundo a Resolução-RDC n. ${ }^{\circ}$ 302, de 13 de outubro de $2005^{13}$, programas que têm como objetivo o controle de qualidade interno e externo, devem ser implementados e executados nos laboratórios clínicos para que sejam evitados os erros dos diagnósticos e as diminuiçóes dos falso-negativos ${ }^{14}$. Os métodos de avaliação recomendados pela Portaria n. ${ }^{\circ} 3.388$, de 30 de dezembro de 2013, que redefinem a qualificação nacional em citopatologia na prevenção do câncer do colo do útero, são chamados de Qualificaçáo Nacional em Citopatologia (QualiCito) para prevençáo do câncer do colo uterino. A QualiCito consiste em estabelecer padróes e avaliar a qualidade do exame de Papanicolaou do colo do útero, monitorando os desempenhos dos prestadores de serviços de laboratórios públicos e privados ao Sistema Único de Saúde. Nesse ano, foi criada a primeira edição do Manual de Gestão da Qualidade para Laboratório de Citopatologia ${ }^{14}$.

Este consiste no monitoramento interno da qualidade (MIQ) e no monitoramento externo da qualidade (MEQ) no intuito de permitir o aprimoramento dos escrutinadores, unificando os critérios citomorfológicos e a melhora da qualidade das amostras a serem analisadas ${ }^{14}$.

$\mathrm{O}$ objetivo deste estudo foi avaliar os indicadores de qualidade, de acordo com o Manual de Gestáo da Qualidade para Laboratório de Citopatologia ${ }^{14}$.

\section{MÉTODO}

Trata-se de um estudo retrospectivo analítico e descritivo, no qual foram verificados os laudos dos exames citopatológicos encaminhados ao Setor de Citopatologia do Laboratório Clínico Pontifícia Universidade Católica de Goiás (LC-PUC-Goiás) no período de janeiro de 2013 a dezembro de 2017. Este trabalho foi aprovado pelo Comitê de Ética em Pesquisa da PUC-Goiás, de acordo com o número de protocolo 235.376, intitulado "Levantamento de doenças transmissíveis e não transmissíveis no laboratório clínico da Pontifícia Universidade Católica de Goiás (PUC-Goiás), Laboratório da Santa Casa de Misericórdia de Goiânia, Laboratório do Hospital da Policial Militar de Goiás e Posto de Coleta da UABSF Vila Mutiráo, Região Noroeste de Goiânia-GO", que proporciona a coleta de dados das amostras dos locais citados.

O resultado do exame citopatológico foi categorizado de acordo com a Nomenclatura Brasileira para Laudos Cervicais e Condutas Preconizadas ${ }^{15}$, baseada nos critérios citopatológicos definidos no Sistema de Bethesda ${ }^{6}$. Os resultados citológicos encontrados foram categorizados de acordo com o grau e a natureza da seguinte maneira: 
células escamosas atípicas de significado indeterminado possivelmente não neoplásicas (ASC-US); células escamosas atípicas de significado indeterminado não podendo excluir lesão intraepitelial de alto grau (ASC-H); lesão intraepitelial escamosa de baixo grau (LSIL); HSIL; células glandulares atípicas (AGC); AIS; carcinoma e adenocarcinoma invasor.

Os dados foram adicionados a um banco de dados no programa Excel 2013. Posteriormente, foram calculados os indicadores de qualidade, seguindo o Manual de Gestão da Qualidade para Laboratório de Citopatologia, conforme o Quadro 1.

\section{RESULTADOS}

Neste estudo, foram avaliados os laudos citológicos do laboratório clínico da PUC-Goiás, durante o período de janeiro 2013 a dezembro 2017. Do total de 6.809 diagnósticos citopatológicos, observaram-se 99,4\% (6.768/6.809) diagnósticos satisfatórios, sendo 91,3\% (6.215/6.809) resultados negativos, 8,1\% (553/6.809) diagnósticos com anormalidades citológicas e 0,6\% (41/6.809) de exames insatisfatórios (Tabela 1).

A Tabela 2 demonstra um total de $8,1 \%$ de anormalidades citológicas (553/6.809). Entre as anormalidades, foram encontradas prevalências de 2,40\% (162/6.809) de ASC-US; 2,80\% (193/6.809) de ASC-H; 1,50\% de LSIL (101/6.809); 0,90\% de HSIL (64/6.809); 0,50\% de AGC (31/6.809); 0,015\% de carcinoma (1/6.809); e 0,015\% de adenocarcinoma (1/6.809).

Os resultados do MIQ estão demonstrados na Tabela 3. O índice de positividade (IP) dos anos 2013 a 2017 estão dentro dos valores esperados, 10,5\%, 7,9\%, 8,6\%,

Quadro 1. Fórmulas e descrições para avaliação dos indicadores de qualidade internos dos exames citopatológicos

\begin{tabular}{|c|c|c|}
\hline $\begin{array}{c}\text { Indicadores de } \\
\text { qualidade }\end{array}$ & Fórmula & Descrição \\
\hline IP & $\begin{array}{l}\text { Número de resultados alterados em } \\
\text { determinado local e período x } 100 \\
\text { Total de exames satisfatórios realizados } \\
\text { no mesmo local e período }\end{array}$ & $\begin{array}{l}\text { Percentual de exames classificados como alterados } \\
\text { (ASC-US; ASC-H; LSIL; HSIL; HSIL não podendo } \\
\text { excluir microinvasão); carcinoma epidermoide } \\
\text { invasor; AGC; AIS, adenocarcinoma invasor, } \\
\text { células atípicas de origem indefinida e outras } \\
\text { neoplasias) entre os satisfatórios. O IP expressa a } \\
\text { prevalência de alterações celulares nos exames e } \\
\text { a sensibilidade do processo do rastreamento em } \\
\text { detectar lesões na população examinada. Deve } \\
\text { ser analisado em conjunto com os indicadores } \\
\text { referentes às atipias de significado indeterminado. } \\
\text { Os resultados do IP são categorizados em: muito } \\
\text { baixo (IP abaixo de } 2 \% \text { ); baixo (IP entre } 2 \% \text { e } 2,9 \% \text { ); } \\
\text { esperado (IP entre } 3 \% \text { e } 10 \% \text { ); acima do esperado } \\
\text { (IP acima de } 10 \%, \text { levando em consideração que } \\
\text { tais prestadores podem atender a serviços de } \\
\text { referência secundária em patologia cervical) }\end{array}$ \\
\hline $\begin{array}{l}\text { Percentual de } \\
\text { exames } \\
\text { compatíveis com } \\
\text { ASC } \\
\text { entre os exames } \\
\text { satisfatórios }\end{array}$ & $\begin{array}{l}\text { Número de exames com ASC-US e } \\
\qquad \text { ASC-H } \times 100 \\
\text { Total de exames satisfatórios }\end{array}$ & $\begin{array}{l}\text { São os casos de dúvida diagnóstica, no qual } \\
\text { os achados citológicos são insuficientes para o } \\
\text { diagnóstico de lesão intraepitelial. Incluem os } \\
\text { casos de ASC-US e ASC-H. Espera-se que no } \\
\text { máximo } 4 \% \text { a } 5 \% \text { de todos dos exames sejam } \\
\text { classificados como ASC }\end{array}$ \\
\hline $\begin{array}{l}\text { Percentual } \\
\text { de exames } \\
\text { compatíveis } \\
\text { com HSIL entre } \\
\text { os exames } \\
\text { satisfatórios }\end{array}$ & $\begin{array}{l}\text { Número de exames HSIL x } 100 \\
\text { Total de exames satisfatórios }\end{array}$ & $\begin{array}{l}\text { Esse indicador mede a capacidade do laboratório } \\
\text { de detectar lesões verdadeiramente precursoras } \\
\text { do câncer do colo do útero; ou seja, as HSIL. Seu } \\
\text { resultado deve ser } \geq 0,4 \%\end{array}$ \\
\hline Razão ASC/SIL & $\begin{array}{l}\text { Número de exames compatíveis com } \\
\text { ASC-US e ASC-H } \\
\text { Número de exames com LSIL e HSIL }\end{array}$ & Seu resultado deve ser $<3$ \\
\hline
\end{tabular}

Legendas: AGC: células glandulares atípicas; AIS: adenocarcinoma in situ; ASC-H: células escamosas atípicas de significado indeterminado não podendo excluir lesão intraepitelial de alto grau; ASC-US: células escamosas atípicas de significado indeterminado possivelmente não neoplásicas; HSIL: lesôes intraepiteliais escamosas de alto grau; IP: índice de positividade; LSIL: lesão intraepitelial escamosa de baixo grau. 
Tabela 1. Prevalência dos resultados dos exames citopatológicos negativos, insatisfatórios e anormalidades citológicas, realizados no laboratório clínico da PUC-Goiás no período de 2013 a 2017

\begin{tabular}{ccccccccc}
\hline \multirow{2}{*}{ Ano } & \multicolumn{2}{c}{ Negativos } & \multicolumn{2}{c}{ Insatisfatórios } & \multicolumn{2}{c}{$\begin{array}{c}\text { Anormalidades } \\
\text { citológicas }\end{array}$} & \multicolumn{2}{c}{ Total } \\
\cline { 2 - 9 } & $\mathbf{n}$ & $\%$ & $\mathbf{n}$ & $\%$ & $\mathbf{n}$ & $\%$ & $\mathbf{n}$ & $\%$ \\
\hline 2013 & 1.622 & 89,2 & 5 & 0,3 & 191 & 10,5 & 1.818 & 100 \\
2014 & 1.547 & 91,4 & 12 & 0,7 & 133 & 7,9 & 1.692 & 100 \\
2015 & 1.035 & 90,2 & 15 & 1,3 & 98 & 8,5 & 1.148 & 100 \\
2016 & 1.007 & 93,2 & 0 & 0 & 74 & 6,8 & 1.081 & 100 \\
2017 & 1.004 & 94,0 & 9 & 0,8 & 57 & 5,2 & 1.070 & 100 \\
Total & 6.215 & 91,3 & 41 & 0,6 & 553 & 8,1 & 6.809 & 100 \\
\hline
\end{tabular}

Tabela 2. Prevalência dos exames citopatológicos realizados no laboratório clínico da PUC-Goiás no período de 2013 a 2017

\begin{tabular}{|c|c|c|c|c|c|c|c|c|c|c|c|c|c|c|c|c|c|c|c|c|}
\hline \multirow{2}{*}{ Ano } & \multicolumn{2}{|c|}{ Negativo } & \multicolumn{2}{|c|}{ Insatisfatório } & \multicolumn{2}{|c|}{ ASC-US } & \multicolumn{2}{|c|}{ ASC-H } & \multicolumn{2}{|c|}{ LSIL } & \multicolumn{2}{|c|}{ HSIL } & \multicolumn{2}{|c|}{$A G C$} & \multicolumn{2}{|c|}{ Carcinoma } & \multicolumn{3}{|c|}{ Adenocarcinoma } & \multirow{2}{*}{\begin{tabular}{|c|} 
Total \\
$\%$
\end{tabular}} \\
\hline & n & $\%$ & n & $\%$ & n & $\%$ & $\mathbf{n}$ & $\%$ & n & $\%$ & n & & n & & n & $\%$ & n & $\%$ & n & \\
\hline & 1.622 & & 5 & & 49 & 2,60 & 76 & & 38 & & 25 & & 3 & & & & & & & 100,00 \\
\hline 2014 & 1.547 & 91,40 & 12 & 0,70 & 32 & 2,00 & 53 & 3,10 & 26 & 1,50 & 18 & 1,1 & 4 & & 0 & & 0 & 0,00 & 1.692 & 100,00 \\
\hline 2015 & 1.035 & 90,20 & 15 & 1,30 & 39 & 3,40 & 31 & 2,70 & 17 & & 0 & & 4 & & 1 & & 0 & 0,00 & & 100,00 \\
\hline 2016 & 1.007 & & 0 & & 24 & & 20 & & 12 & & 7 & & & & & & 1 & & & \\
\hline 2017 & 1.004 & 94,00 & 9 & 0,80 & 18 & 1,70 & 13 & 1,20 & 8 & & 8 & & 10 & & 0 & & 0 & & 1.070 & 100,00 \\
\hline Total & 6.215 & 91,30 & 41 & 0,60 & 162 & 2,40 & 193 & 2,80 & 101 & 1,50 & 64 & 0,90 & 31 & 0,50 & 1 & 0,015 & 1 & 0,015 & 6.809 & 100,00 \\
\hline
\end{tabular}

Legendas: ASC-US: células escamosas atípicas de significado indeterminado possivelmente não neoplásicas; ASC-H: células escamosas atípicas de significado indeterminado não podendo excluir lesão intraepitelial de alto grau; LSIL: lesão intraepitelial escamosa de baixo grau; HSIL: lesão intraepitelial escamosa de alto grau; AGC: células glandulares atípicas.

Tabela 3. Indicadores de qualidade internos dos exames citopatológicos realizados no laboratório clínico da PUC-Goiás em relação ao índice de positividade, percentual de exames compatíveis com ASC entre os exames satisfatórios e razão de ASC/SIL no período de 2013 a 2017

\begin{tabular}{lcccc}
\hline Ano & Índice de positividade & \% Compatíveis com HSIL & \% ASC satisfatórios & Razão ASC/SIL \\
\hline 2013 & $10,5 \%$ & $1,3 \%$ & $6,8 \%$ & 1,9 \\
2014 & $7,9 \%$ & $1,0 \%$ & $5,0 \%$ & 1,9 \\
2015 & $8,6 \%$ & $0,5 \%$ & $6,1 \%$ & 3,0 \\
2016 & $6,8 \%$ & $0,6 \%$ & $4,0 \%$ & 2,3 \\
2017 & $5,3 \%$ & $0,7 \%$ & $2,9 \%$ & 1,9 \\
\hline
\end{tabular}

Legendas: HSIL: lesão intraepitelial escamosa de alto grau; ASC: células escamosas atípicas; SIL: lesão intraepitelial escamosa de baixo grau.

$6,8 \%$ e $5,3 \%$, respectivamente. Somente no ano de 2013 , foi observado um pouco acima do que é recomendado para os laboratórios que prestam serviços para o Sistema Único de Saúde (SUS). A porcentagem de compatíveis com HSIL apresentou-se da seguinte maneira, no ano de 2013, 1,3\%; 2014, 1,0\%; 2015, 0,5\%; 2016, $0,6 \%$; e $2017,0,7 \%$. Esses valores estão dentro do que é estabelecido pelos índices de rastreamento $\geq 0,4 \%$. A relaçáo de ASC/satisfatórios demostrou valores superiores aos estabelecidos, nos anos de 2013 com 6,8\% e 2015 com $6,1 \%$. Segundo o Manual de gestão para Controle de
Qualidade $^{14}$, espera-se que, no máximo, $4 \%$ a $5 \%$ de todos dos exames sejam classificados como ASC, valores que ultrapassam $5 \%$ necessitam de uma atenção diferenciada.

\section{DISCUSSÃO}

A avaliaçáo da qualidade dos exames analisados no laboratório de análises clínicas da PUC-Goiás se deu por análise de quatro indicadores, de acordo o Manual de Gestão da Qualidade para Laboratório de Citopatologia ${ }^{14}$. Em relação ao IP, que determina a prevalência de alterações 
celulares e indica a sensibilidade do exame no rastreamento de lesóes, no período de 2013 a 2017, foi de 7,8\%. Esse resultado encontra-se dentro dos valores esperados e recomendados, que é de 3\% a 10\%, indicando um bom desempenho do processo de triagem para detecção de lesóes precursoras do câncer do colo do útero. No estudo de Plewka et al. ${ }^{16}$, foi observado um IP de 5,1\% em um laboratório no Estado do Maranhão no período de janeiro de 2010 a dezembro de 2012.

No estudo realizado por Paula et al. ${ }^{17}$, no laboratório de análises clínicas da PUC-Goiás, foram encontrados, durante o período de 2009 a 2013, IP de 5\%, 4\%, 7\%, 7\% e $11 \%$, respectivamente, com a média dos anos avaliados de $7 \%$. No mesmo estudo, destaca-se a concordância com os resultados dos relatórios do MEQ, variando em $85,6 \%, 87,2 \%, 95,9 \%, 86,9 \%$ e 90,6\%. De fato, o MEQ é fundamental para garantir a melhoria contínua da qualidade dos exames citológicos.

O percentual de HSIL avalia a capacidade do laboratório de detectar lesóes precursoras do câncer do colo do útero, na tentativa de diminuir sua incidência e mortalidade $^{12}$. Neste estudo, no período avaliado, HSIL obteve um percentual de 0,9\%, mostrando-se dentro do valor preconizado que é $\geq 0,4 \%$. Segundo Bortolon et al. $^{18}$, o percentual de HSIL, para as grandes Regióes e para o Brasil, ficou abaixo de 0,5\%, entre os Estados, apenas Roraima e Distrito Federal apresentaram resultados acima do estabelecido. De fato, a correta identificação do HSIL, em combinação com a confirmação diagnóstica, tratamento e acompanhamento adequado, pode impedir a evolução de lesão ao câncer do colo do útero ${ }^{19,20}$.

A média de ASC/satisfatórios, durante o período avaliado, foi de 5,0\%. Esse valor está condizente com o estabelecido, que é um percentual de até 5\%. Esse índice compóe uma situação de dúvida diagnóstica, já que o diagnóstico de ASC é mais variável do que o diagnóstico de SIL de alto e baixo graus ${ }^{14}$. Bortolon et al. ${ }^{18}$ observaram um percentual de ASC entre os exames satisfatórios de 1,1\% nos laboratórios do Paraná, 1,1\% no Rio Grande do Sul e 1,3\% em Santa Catarina, todos abaixo da média.

A razão de ASC/SIL revela que as porcentagens dos anos avaliados estáo dentro do preconizado, $<3$, podendo sugerir que, neste estudo, não houve intercorrência com a parte técnica das análises das amostras citológicas, entretanto a educação continuada não pode ser descartada, já que o aperfeiçoamento garante a qualidade dos diagnósticos citopatológicos ${ }^{14}$.

A qualidade da amostra tem um papel fundamental no resultado diagnóstico das lesóes precursoras do câncer do colo uterino ${ }^{21-24}$. Muitos fatores que podem reduzir a precisão do exame citopatológico para o diagnóstico das lesóes precursoras e o câncer do colo uterino são os considerados obscurecedores, prejudicando parcialmente e/ou totalmente os esfregaços, tais como: sangue, infiltrado leucocitário, áreas espessas, dessecamento, artefatos de estiramento, lubrificante e contaminação, segundo indicado no Sistema de Bethesda ${ }^{6}$. Além da não representatividade da zona de transformação. Esses problemas ocorrem no momento da coleta que resultam em resultados falso-negativos ${ }^{14}$.

O total de resultados citopatolólgicos, 99,4\%, foi de diagnósticos satisfatórios; destes, 91,3\% foram diagnosticados negativos e 8,1\%, diagnósticos com anormalidades citológicas. Em relação à prevalência de insatisfatórios, foi de $0,6 \%$ com maior percentual no ano de 2015, mostrando 1,5\%. Entre as anormalidades, foram encontradas prevalências de 2,40\% de ASC-US; 2,80\% de ASC-H; 1,50\% de LSIL; 0,90\% de HSIL; 0,50\% de AGC; 0,015\% de carcinoma (1/6.809); e $0,015 \%$ de adenocarcinoma (1/6.809). Esses resultados de carcinomas e adenocarcinomas terem sido pouco expressivos podem ser explicados pelo fato do período analisado e de se tratar de resultados de uma populaçáo que realiza o exame citopatológico com a finalidade de rastreamento.

O MIQ nos laboratórios de citopatologia ${ }^{14}$ é importante, uma vez que podem ocorrer erros no momento do escrutínio. Alguns motivos levam à subavaliação diagnóstica das células neoplásicas que estão presentes no esfregaço, porém não são reconhecidas pelo escrutinador ${ }^{25,26}$. A falta de atenção e concentração, o tempo precário para a análise da lâmina, cansaço mental, sobrecarga do tempo de trabalho e a falta da experiência profissional contribuem para que isso aconteça ${ }^{27}$, o que reforça a necessidade do MIQ nos laboratórios de citopatologia $^{14,20}$.

\section{CONCLUSÃO}

Em razão de o método da citologia do colo útero ser subjetivo, a implementaçáo do controle interno de qualidade deve ser efetiva, utilizando os métodos padronizados, e os indicadores de qualidade devem ser sempre avaliados e interpretados por toda equipe do laboratório. Os indicadores de qualidade são uma ferramenta que indica a necessidade de aprimorar os critérios citomorfológicos entre os escrutinadores.

Contudo, é de suma importância a educação continuada dos profissionais que participam de todas as etapas do processo, da fase pré-analítica à analítica, para que possíveis erros possam ser corrigidos e medidas preventivas tomadas para uma melhor qualidade na interpretação dos exames citopatológicos. 


\section{CONTRIBUIÇÕES}

Mackson Jardel Silva Santos contribuiu com o planejamento do estudo, obtenção, análise e interpretação dos dados e redação e revisão crítica do texto. Andrea Alves Ribeiro contribuiu com o planejamento do estudo, orientação da obtenção, análise e interpretação dos dados e redação e revisão crítica do texto. Ambos aprovaram a versão final a ser publicada.

\section{DECLARAÇÃO DE CONFLITOS DE INTERESSE}

Nada a declarar.

\section{FONTES DE FINANCIAMENTO}

Não há.

\section{REFERÊNCIAS}

1. INCA: Instituto Nacional de Câncer José Alencar Gomes da Silva [Internet]. Rio de Janeiro: INCA; [data desconhecida]. Tipos de câncer: câncer do colo do útero; [acesso 2018 jan. 29]; [1 tela]. Disponível em: https://www. inca.gov.br/tipos-de-cancer/cancer-do-colo-do-utero

2. Walboomers JM, Jacobs MV, Manos MM, et al. Human papillomavirus is a necessary cause of invasive cervical cancer worldwide. J Pathol. 1999;189(1):129. doi: https://doi.org/10.1002/(SICI)10969896(199909) 189:1<12::AID-PATH431>3.0.CO;2-F

3. Doorbar J, Quint W, Banks L, et al. The biology and life-cycle of human papillomaviruses. Vaccine. 2012;30(Suppl 5):F55-70. doi: https://doi.org/10.1016/j. vaccine.2012.06.083

4. Ribeiro AA, Costa MC, Alves RRF, et al. HPV infection and cervical neoplasia: associated risk factors. Infect Agent Cancer. 2015;10:16. doi: https://doi.org/10.1186/ s13027-015-0011-3

5. Mendonça FC, Costa GO, Ribeiro AA. Prevalência do diagnóstico citopatológico: uma abordagem sobre as condutas preconizadas pelo Ministério da Saúde. Estudos (Goiânia). 2019;46:17-23. doi: http://dx.doi. org/10.18224/evs.v46i1.6453

6. Nayar R, Wilbur DC, editores. Sistema Bethesda para relato de citologia cervical: definiçôes, critérios e notas explicativas. 3.ed. São Paulo: Livromed Paulista; 2018.

7. World Health Organization. Cancer control: knowledge into action: WHO guide for effective programmers [Internet]. Geneva: WHO; 2007. [cited 2018 Mar 21]. 56 p. (module; 2). Available from: www.who.int/cancer/ modules/Prevention\%20Module.pdf

8. Bergeron C, Masseroli M, Ghezi A, et al. Quality control of cervical cytology in high-risk women.
PAPNET system compared with manual rescreening. Acta Cytol. 2000;44(2):151-7. doi: http://dx.doi. org/10.1159/000326353

9. Ferraz MGMC, Dall'Agnol M, di Loreto C, et al. 100\% rapid rescreening for quality assurance in a quality control program in a public health cytologic laboratory. Acta Cytologica. 2005;49(6):639-43. doi: https://doi. org/10.1159/000326252

10. Renshaw AA. An accurate and precise methodology for routine determination of the false-negative rate of Papanicolaou smear screening. Cancer Cytopatho. 2001;93(2):86-92. doi: https://doi.org/10.1002/ cncr.9012

11. Halford JA, Wright RG, Ditchmen EJ. Quality assurance in cervical cytology screening. Comparison of rapid rescreening and the PAPNET testing system. Acta Cytol. 1997;41(1):79-81. doi: https://doi. org/10.1159/000332309

12. Pias AA, Vargas VRA. Avaliaçáo dos exames citológicos de Papanicolaou com células epiteliais atípicas e respectivos exames colposcópicos com relação aos exames histopatológicos. Rev Bras Anal Clin. 2009;41(2):155-160.

13. Agência Nacional de Vigilância Sanitária. ResoluçãoRDC no 302, de 13 de outubro de 2005. Diário Oficial da Uniāo, Brasília, DF; 2005 out. 14. Seção 1, p. 33.

14. Instituto Nacional de Câncer José Alencar Gomes da Silva. Manual de gestão da qualidade para laboratório de citopatologia. 2. ed. Rio de Janeiro: INCA; 2016. 160 p.

15. Instituto Nacional de Câncer. Nomenclatura brasileira para laudos cervicais e condutas preconizadas: recomendaçôes para profissionais de saúde. Rio de Janeiro: INCA; 2006.

16. Plewka J, Turkiewicz M, Duarte BF, et al. Avaliação dos indicadores de qualidade de laboratórios de citopatologia cervical. Rev Inst Adolfo Lutz. 2014;73(2):140-147. doi: https://doi.org/10.18241/0073-98552014731599

17. Paula AC, Souza NG, Prado TC, et al. Indicadores do monitoramento interno da qualidade dos exames citopatológicos do Laboratório Clínico da Pontifícia Universidade Católica de Goiás (PUC-GO). Rev Bras Anal Clin. 2017;49(2):200-5. doi: https://doi. org/10.21877/2448-3877.201700534

18. Bortolon PC, Silva MAF, Corrêa FM, et al. Avaliação da qualidade dos laboratórios de citopatologia do colo do útero no Brasil. Rev Bras Cancerol. 2012;58(3):435-44.

19. McGraw SL, Ferrante JM. Update on prevention and screening of cervical cancer. World J Clin Oncol. 2014;5(4):744-52. doi: https://doi.org/10.5306/wjco. v5.14.744

20. Cardoso Filho LI, Tavares SBN, Siqueira MLB, et al. Internal quality control indicators in cervical cytopathology of a university laboratory. Cytopathology. 2018;29(4):355-60. doi: https://doi.org/10.1111/ cyt. 12571 
21. Amaral RG, Ribeiro AA, Miranda FA, et al. Fatores que podem comprometer a qualidade dos exames citopatológicos no rastreamento do câncer do colo do útero. Rev Bras Anal Clin. 2006;38(1):3-6.

22. Thuler LCS, Aguiar SS, Bergmann A. Determinantes do diagnóstico em estadio avançado do câncer do colo do útero no Brasil. Rev Bras Ginecol Obstet. 2014;36(6):237-43. doi: http://dx.doi.org/10.1590/ S0100-720320140005010

23. Pajtler M, Audy-Jurković S, Škopljanac-Mačina L, et al. Rapid cervicovaginal smear screening: method of quality control and assessing individual cytotechnologist performance. Cytopathology. 2006;17(3):121-6. doi: http://dx.doi.org/10.1111/j.1365-2303.2006.00367.x

24. Zhao L, Wentzensen N, Zhang RR, et al. Factors associated with reduced accuracy in papanicolaou tests for patients with invasive cervical cancer. Cancer Cytopathol. 2014;122(9):694-701. doi: https://doi.org/10.1002/ cncy. 21443

25. Vecchione A, Cenci M. The false negative smears: facts and solutions. In: Testa R, Jakob CA, Huguet JO, editors. Proceedings of the X World Congress of Cervical Pathology \& Colposcopy; 1999 Nov 7-11; Buenos Aires, Argentina, Bologna: Italy; 1999:53-57.

26. Pittoli JE, Mello ES, Pereira SMM, et al. Revisão de esfregaços cervicais negativos em pacientes com lesóes intra-epiteliais de alto grau. J Bras Patol Med Lab. 2003;39(3):219-21. doi: http://dx.doi.org/10.1590/ S1676-24442003000300008

27. Queiroz FN. A importância da enfermagem na prevençáo do câncer de colo uterino. [monografia]. Batatais (SP): Centro Universitário Claretiano; 2006. 67p. 